\title{
Diversity of Cercopithifilaria species in dogs from Portugal
}

\author{
Helder CE Cortes ${ }^{1}$, Luís Cardoso ${ }^{2,3^{*}}$, Alessio Giannelli ${ }^{4}$, Maria Stefania Latrofa ${ }^{4}$, Filipe Dantas-Torres ${ }^{4,5}$ \\ and Domenico Otranto ${ }^{4}$
}

\begin{abstract}
Background: Filarioids belonging to the genus Cercopithifilaria (Spirurida: Onchocercidae) have been described in dogs in association with Rhipicephalus sanguineus group ticks, which act as their biological vectors. This study represents the first investigation on Cercopithifilaria spp. in dogs from Portugal.

Findings: Dogs $(n=102)$ from the Algarve region (south of Portugal) were sampled by skin snip collection and tissues were left to soak overnight in saline solution. Sediments were observed under a light microscope and the detected microfilariae identified according to their morphology. Twenty-four dogs (23.5\%) were found infected with at least one species of Cercopithifilaria, namely C. bainae (9.8\%), C. grassii (3.9\%) and Cercopithifilaria sp. II sensu Otranto et al., 2013 (13.7\%). Results were confirmed by molecular amplification of partial cytochrome $c$ oxidase subunit I and 125 rRNA genes and sequence analysis. Co-infections with more than one Cercopithifilaria species were detected in $3.9 \%$ of the animals.

Conclusions: This is the first report of Cercopithifilaria spp. in dogs from Portugal. The estimated level of infection with C. bainae, C. grassii and Cercopithifilaria sp. II suggests that these filarioids are prevalent in the canine population of southern Portugal.
\end{abstract}

Keywords: Cercopithifilaria bainae, Cercopithifilaria grassii, Cercopithifilaria sp. II, Dogs, Portugal

\section{Findings}

Several species of vector-borne filarial nematodes (Spirurida: Onchocercidae) have been found parasitizing dogs in Europe. In particular, Dirofilaria immitis, also known as the canine heartworm, and Dirofilaria repens are the agents of cardiopulmonary and subcutaneous dirofilarioses, respectively, which represent diseases of veterinary importance and zoonotic potential [1]. These two species, together with Acanthocheilonema dracunculoides and Acanthocheilonema reconditum, have microfilariae that circulate in the bloodstream. However, dogs may also be infected with other less well-known filarial species, such as Onchocerca lupi and Cercopithifilaria spp., whose microfilariae are found in dermal tissues $[2,3]$.

\footnotetext{
* Correspondence: Icardoso@utad.pt

${ }^{2}$ Department of Veterinary Sciences, School of Agrarian and Veterinary Sciences, University of Trás-os-Montes e Alto Douro (UTAD), Vila Real, Portugal

${ }^{3}$ Parasite Disease Group, Instituto de Biologia Molecular e Celular, Universidade do Porto, Oporto, Portugal

Full list of author information is available at the end of the article
}

The genus Cercopithifilaria, originally described as a subgenus of Dipetalonema by Eberhard [4], currently comprises 28 species that are parasites of a broad range of wild and domestic mammals and are transmitted by ixodid ticks [5-7]. Adult worms usually dwell beneath the cutaneous tissues, where they are not easily detected, and their microfilariae are found exclusively in the dermis [5]. Filarioids of the genus Cercopithifilaria have been described in dogs from Brazil and Europe [6,8]. Up until now, Cercopithifilaria grassii, Cercopithifilaria bainae and Cercopithifilaria sp. II sensu Otranto et al., 2013 have been morphologically and molecularly differentiated in canine populations in Europe [7,9-11]. Microfilariae of these three species can be distinguished from each other by their length and general morphological features and also by the inter-specific genetic distance of the mitochondrial cytochrome $c$ oxidase subunit I $(\operatorname{cox} 1)$ and $12 S$ rRNA genes $[6,10]$.

The field distribution of C. bainae overlaps with that of Rhipicephalus sanguineus group ticks [12], which act as vectors for this nematode [2], as experimentally 
demonstrated [13,14]. C. bainae has been found in dogs and ticks from Italy, Spain and Greece [2,15], in one dog from Romania [11] and also in ticks from Portugal [16]; M.S. Latrofa, F. Dantas-Torres, A. Giannelli and D. Otranto, personal communication.

Infections with canine filarioids, such as $D$. immitis, A. dracunculoides, A. reconditum and O. lupi, have previously been identified in Portugal [17-19], but no information on Cercopithifilaria spp. is available from dogs. In order to fill this gap of knowledge, in November 2012, 102 dogs (65 females and 37 males) housed in two shelters located in center-eastern areas of the Algarve region (the southernmost part of mainland Portugal) were sampled. The animals were estimated to be 1-3 years $(n=19)$, 4-8 years $(n=78), 9-11$ years $(n=4)$ or 12-15 years old $(\mathrm{n}=1)$. An informed consent for inclusion of dogs in this survey was obtained from the owner before sampling. The study design and experimental procedures were approved by the ethical commission of the University of Évora as complying with the Portuguese legislation for the protection of animals (Law no. 92/1995). Skin snips of around $0.01 \mathrm{~cm}^{3}(0.2 \times 0.2 \times 0.2 \mathrm{~cm})$ were collected from the forehead region using a disposable scalpel and soaked in saline solution ( $\mathrm{pH}$ 7.4) for $12 \mathrm{~h}$ in an incubator at $30^{\circ} \mathrm{C}$. Dermal microfilariae were searched for in $20 \mu \mathrm{l}$ sediment placed on a glass slide, using a light microscope (magnifications of $\times 40$ and $\times 100$ ), and identified according to their morphological features, as previously described $[6,10]$. Microfilariae were photographed with a digital camera mounted on the microscope (DP20, Olympus, Japan) and measurements (in micrometers) taken with the Live Cell Imaging software (Olympus).

Following morphological analysis of microfilariae, sediments scoring positive were stored in $70 \%$ ethanol and species identification was subsequently assessed by PCR amplification and DNA sequencing $[6,10]$. Microfilariae were isolated and genomic DNA extracted from individual specimens using a commercial kit (DNeasy Blood \& Tissue Kit, Qiagen, Germany) in accordance with the manufacturer's instructions. Samples were molecularly processed for specific amplification of cox 1 and 12S rRNA gene fragments ( $\sim 889$ and $\sim 330$ bp in size, respectively), with primers and procedures as described elsewhere [6].

Amplicons were purified using Ultrafree-DA columns (Amicon, Millipore, USA) and sequenced directly with the Taq DyeDeoxyTerminator Cycle Sequencing Kit (v.2, Applied Biosystems, USA) in an automated sequencer (ABI-PRISM 377, Applied Biosystems). The sequences were determined from both strands, using the same primers individually as for PCR, and the electropherograms verified by eye. The sequences were aligned using ClustalW program and compared among them and with those available in GenBank dataset by Basic Local Alignment Search Tool (BLAST - http://blast.ncbi.nlm.nih.gov/Blast.cgi).

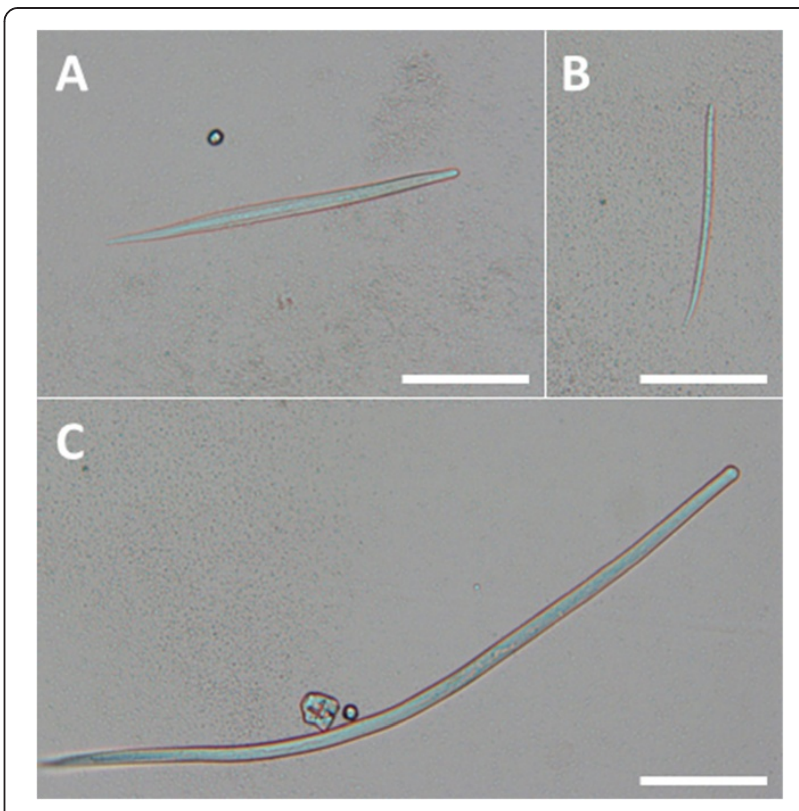

Figure 1 Microfilariae of: (A) Cercopithifilaria sp. II; (B) Cercopithifilaria bainae; and (C) Cercopithifilaria grassii. Sediment of forehead skin snips from dogs (scale-bar $=100 \mu \mathrm{m}$ ).

For statistical analysis, the Chi-squared or Fisher's exact tests compared proportions of positivity and a $p$ value $<0.05$ was considered as statistically significant [20]. The exact binomial test estimated confidence intervals (CI) for proportions, with a 95\% confidence level. Analyses were done using StatLib or SPSS 21 software for Windows.

By microscopy, microfilariae of C. bainae, C. grassii and Cercopithifilaria sp. II were detected (Figure 1), with an individual prevalence of 9.8\% (CI: $4.8-17.3 \%$ ), 3.9\% (CI: $1.1-9.7 \%$ ) and $13.7 \%$ (CI: 7.0-20.8\%), respectively, both in single or in mixed infections. The prevalence of Cercopithifilaria sp. II was significantly higher than that

\begin{tabular}{|c|c|c|c|}
\hline Cercopithifilaria spp. & $\begin{array}{l}\text { No. of positive } \\
\text { dogs }\end{array}$ & $\%$ & $\mathrm{Cl}(\%)$ \\
\hline Single infections & 20 & $19.6^{\mathrm{a}}$ & $12.4-28.6$ \\
\hline Cercopithifilaria bainae & 7 & 6.9 & $2.4-11.6$ \\
\hline Cercopithifilaria grassii & 3 & 2.9 & $0.6-8.3$ \\
\hline Cercopithifilaria sp. II & 10 & 9.8 & $4.8-17.3$ \\
\hline Mixed infections & 4 & $3.9^{\mathrm{a}}$ & $1.1-9.7$ \\
\hline C. bainae + Cercopithifilaria sp. II & 3 & 2.9 & $0.6-8.3$ \\
\hline C. grassii + Cercopithifilaria sp. II & 1 & 1.0 & $0.02-5.3$ \\
\hline Total & 24 & 23.5 & 15.7-33.0 \\
\hline
\end{tabular}

Cl: confidence interval; ${ }^{\mathrm{a}}: p<0.001$. 
of $C$. grassii ( $p=0.023$ ), but no differences were found in pairwise comparison between $C$. bainae and C. grassii or C. bainae and Cercopithifilaria sp. II. The prevalence of single infections $(19.6 \%)$ was significantly higher $(p<0.001)$ than that of mixed infections (3.9\%) (Table 1). Differences in the prevalence of infection with at least one of the three Cercopithifilaria species were not statistically significant between female (24.6\%) and male dogs $(21.6 \%)$ or between age groups, i.e. $1-3$ years $(31.6 \%), 4-8$ years $(21.8 \%), 9-11$ years $(25.0 \%)$ and $12-15$ years $(0.0 \%)$.

Microfilariae of $C$. bainae $(\mathrm{n}=10)$ had an average length \pm standard deviation (SD) of $181.1 \pm 7.5 \mu \mathrm{m}$ (range: 170.1-196.4 $\mu \mathrm{m}$ ). The average length $\pm \mathrm{SD}$ of C. grassii microfilariae $(\mathrm{n}=6)$ was $630.8 \pm 13.8 \mu \mathrm{m}$ (range: 610.1-643.5 $\mu \mathrm{m}$ ). Microfilariae of Cercopithifilaria sp. II $(\mathrm{n}=13)$ had an average length \pm SD of $277.0 \pm$ $15.5 \mu \mathrm{m}$ (range: $260.9-307.4 \mu \mathrm{m}$ ).

The BLAST analysis revealed 99\% homology of the amplified sequences with the closest accession numbers of C. bainae (JF461457 and JF461461), C. grassii (JQ837810 and JQ837812) and Cercopithifilaria sp. II (JQ837809 and JQ837811) available in GenBank database (accessions displayed for cox 1 and 12S rRNA genes, respectively).

Morphological and molecular data concurred in indicating that the dermal microfilariae detected in dogs from southern Portugal belong to three distinct known species of Cercopithifilaria. Infections with C. bainae and Cercopithifilaria sp. II were detected in dogs from the Italian regions of Basilicata [10] and Sardinia [15], whereas C. bainae and C. grassii were detected in Sicily [10]. Sympatric C. bainae, C. grassi and Cercopithifilaria sp. II populations were found in dogs from central Spain [10]. The $23.5 \%$ overall prevalence of infection with at least one of those species (Table 1) is in line with that of $21.6 \%$ found by microscopy in dogs from central Spain [10]. In Italy, the prevalence of Cercopithifilaria spp. has been found to range from $9.4 \%$ in Sardinia [15] to $13.3 \%$ in Sicily [10]. Still by microscopic examination of skin snip sediments, the prevalence of C. bainae in northeastern Greece was 4.3\% [2].

Dogs found infected with Cercopithifilaria spp. did not present physical abnormalities that could be associated to the presence of microfilariae in the skin (data not shown). However, based on clinical and histological findings, a pruritic, diffuse and erythematous dermatitis without any other apparent cause was previously described in two dogs infected with microfilariae of C. bainae [21] or Cercopithifilaria sp. II [10].

In conclusion, the present study highlights that Cercopithifilaria spp. should be considered among those parasites that can cause dermal microfilariae infection in dogs in Portugal. In the meantime, further studies should be carried out in order to elucidate the pathogenic relevance of these filarioids for dogs and other potential hosts.
Competing interests

The authors declare that they have no competing interests.

\section{Authors' contributions}

HCEC designed and supervised the study, collected samples, analyzed data, assisted in drafting and reviewed the manuscript; LC collected samples, analyzed data and wrote the manuscript; AG performed morphometric assessments and reviewed the manuscript; MSL performed DNA extraction and molecular analyses and reviewed the manuscript; FDT analyzed data and reviewed the manuscript; DO planned and supervised the study, analyzed data and reviewed the manuscript. All authors read and approved the final manuscript.

\section{Acknowledgements}

The authors would like to express their gratitude to Riccardo Paolo Lia (University of Bari), for laboratory support, and to Giada Annoscia (University of Bari), for sequence edition.

Publication costs have been supported by Bayer HealthCare-Animal Health Division.

\section{Author details}

${ }^{1}$ Victor Caeiro Laboratory of Parasitology, Instituto de Ciências Agrárias e Ambientais Mediterrânicas, University of Évora, Évora, Portugal. ${ }^{2}$ Department of Veterinary Sciences, School of Agrarian and Veterinary Sciences, University of Trás-os-Montes e Alto Douro (UTAD), Vila Real, Portugal. ${ }^{3}$ Parasite Disease Group, Instituto de Biologia Molecular e Celular, Universidade do Porto, Oporto, Portugal. ${ }^{4}$ Department of Veterinary Medicine, University of Bari, Valenzano, Italy. ${ }^{5}$ Department of Immunology, Centro de Pesquisas Aggeu Magalhães (Fiocruz), Recife, Brazil.

Received: 24 April 2014 Accepted: 29 May 2014

Published: 5 June 2014

\section{References}

1. Otranto D, Dantas-Torres F, Brianti E, Traversa D, Petrić D, Genchi C, Capelli G: Vector-borne helminths of dogs and humans in Europe. Parasit Vectors 2013, 6:16.

2. Otranto D, Brianti E, Latrofa MS, Annoscia G, Weigl S, Lia RP, Gaglio G, Napoli E, Giannetto S, Papadopoulos E, Mirò G, Dantas-Torres F, Bain O: On a Cercopithifilaria sp. transmitted by Rhipicephalus sanguineus: a neglected, but widespread filarioid of dogs. Parasit Vectors 2012, 5:1.

3. Mutafchiev Y, Dantas-Torres F, Giannelli A, Abramo F, Papadopoulos E, Cardoso L, Cortes H, Otranto D: Redescription of Onchocerca lupi (Spirurida: Onchocercidae) with histopathological observations. Parasit Vectors 2013, 6:309.

4. Eberhard ML: Dipetalonema (Cercopithifilaria) kenyensis subgen. et sp. $\mathrm{n}$. (Nematoda: Filarioidea) from African baboons, Papio anubis. J Parasitol 1980, 66:551-554

5. Bain O, Uni S, Takaoka H: A synthetic look at a twenty years old taxon, Cercopithifilaria its probable evolution. In Proceedings of the 10th International Congress of Parasitology (ICOPA X). Vancouver: Monduzzi Editore; 2002:365-368.

6. Otranto D, Brianti E, Dantas-Torres F, Weigl S, Latrofa MS, Gaglio G, Cauquil L, Giannetto S, Bain O: Morphological and molecular data on the dermal microfilariae of a species of Cercopithifilaria from a dog in Sicily. Vet Parasitol 2011, 182:221-229.

7. Otranto D, Varcasia A, Solinas C, Scala A, Brianti E, Dantas-Torres F, Annoscia G, Martin C, Mutafchiev Y, Bain O: Redescription of Cercopithifilaria bainae Almeida \& Vicente, 1984 (Spirurida, Onchocercidae) from a dog in Sardinia, Italy. Parasit Vectors 2013, 6:132.

8. Almeida GLG, Vicente JJ: Cercopithifilaria bainae sp. n. parasita de Canis familiaris (L.) (Nematoda, Filarioidea). Atas Soc Biol Rio Janeiro 1984, 24:18.

9. Noè G: Contribuzioni alla sistematica e alla anatomia del genere Filaria. La Filaria grassii. In . Rome: Istituto di Anatomia Comparata della Regia Università di Roma; 1907:236-252.

10. Otranto D, Brianti E, Dantas-Torres F, Miró G, Latrofa MS, Mutafchiev Y, Bain O: Species diversity of dermal microfilariae of the genus Cercopithifilaria infesting dogs in the Mediterranean region. Parasitology 2013, 140:99-108.

11. Ionică AM, D'Amico G, Mitková B, Kalmár Z, Annoscia G, Otranto D, Modrý D, Mihalca AD: First report of Cercopithifilaria spp. in dogs from 
Eastern Europe with an overview of their geographic distribution in Europe. Parasitol Res, in press.

12. Dantas-Torres F, Latrofa MS, Annoscia G, Giannelli A, Parisi A, Otranto D: Morphological and genetic diversity of Rhipicephalus sanguineus sensu lato from the New and Old Worlds. Parasit Vectors 2013, 6:213.

13. Brianti E, Otranto D, Dantas-Torres F, Weigl S, Latrofa MS, Gaglio G, Napoli E, Brucato G, Cauquil L, Giannetto S, Bain O: Rhipicephalus sanguineus (Ixodida, Ixodidae) as intermediate host of a canine neglected filarial species with dermal microfilariae. Vet Parasitol 2012, 183:330-337.

14. Ramos RAN, Giannelli A, Brianti E, Annoscia G, Cantacessi C, Dantas-Torres F, Otranto D: Tick vectors of Cercopithifilaria bainae in dogs: Rhipicephalus sanguineus sensu lato versus Ixodes ricinus. Parasitol Res 2013, 112:3013-3017.

15. Solinas C, Varcasia A, Brianti E, Giannetto S, Pipia AP, Columbano N, Tosciri G, Dantas-Torres F, Garippa G, Otranto D, Scala A: Cercopithifilaria spp. in dogs in Sardinia Island (Italy). Parasitol Res 2014, 113:675-679.

16. Maia C, Ferreira A, Nunes M, Vieira ML, Campino L, Cardoso L: Molecular detection of bacterial and parasitic pathogens in hard ticks from Portugal. Ticks Tick Borne Dis 2014, 5:409-414.

17. Menn B, Lorentz S, Naucke TJ: Imported and travelling dogs as carriers of canine vector-borne pathogens in Germany. Parasit Vectors 2010, 3:34.

18. Cardoso L, Mendão C, Madeira de Carvalho L: Prevalence of Dirofilaria immitis, Ehrlichia canis, Borrelia burgdorferi sensu lato, Anaplasma spp. and Leishmania infantum in apparently healthy and CVBD-suspect dogs in Portugal - a national serological study. Parasit Vectors 2012, 5:62.

19. Otranto D, Dantas-Torres F, Giannelli A, Latrofa MS, Papadopoulos E, Cardoso L, Cortes H: Zoonotic Onchocerca lupi infection in dogs, Greece and Portugal, 2011-2012. Emerg Infect Dis 2013, 19:2000-2003.

20. Petrie A, Watson P: Statistics for Veterinary and Animal Science. 2nd edition. Oxford: Blackwell Publishing; 2006.

21. Otranto D, Brianti E, Abramo F, Gaglio G, Napoli E, Latrofa MS, Ramos RA, Dantas-Torres F, Bain O: Cutaneous distribution and localization of Cercopithifilaria sp. microfilariae in dogs. Vet Parasitol 2012, 190:143-150.

doi:10.1186/1756-3305-7-261

Cite this article as: Cortes et al:: Diversity of Cercopithifilaria species in dogs from Portugal. Parasites \& Vectors 2014 7:261.

\section{Submit your next manuscript to BioMed Central and take full advantage of:}

- Convenient online submission

- Thorough peer review

- No space constraints or color figure charges

- Immediate publication on acceptance

- Inclusion in PubMed, CAS, Scopus and Google Scholar

- Research which is freely available for redistribution 